\title{
An ALM model for pension funds using integrated chance constraints
}

\author{
Willem K. Klein Haneveld • Matthijs H. Streutker • \\ Maarten H. van der Vlerk
}

Published online: 30 July 2009

(C) The Author(s) 2009. This article is published with open access at Springerlink.com

\begin{abstract}
We discuss integrated chance constraints in their role of short-term risk constraints in a strategic ALM model for Dutch pension funds. The problem is set up as a multistage recourse model, with special attention for modeling short-term risk prompted by the development of new guidelines by the regulating authority for Dutch pension funds. The paper concludes with a numerical illustration of the importance of such short-term risk constraints.
\end{abstract}

Keywords Modeling A ALM - Integrated chance constraints · Multistage mixed-integer recourse

\section{Introduction}

The strategic Asset Liability Management (ALM) problem for pension funds is a dynamic decision problem under uncertainty. Management of assets involves decisions on the strategic portfolio mix, and the liabilities - consisting of future pension payments-depend on indexation policies (correction for inflation). Because of the long time horizon over which the liabilities range, typically in the order of 30 years, the problem is inherently dynamic. Moreover, uncertainty plays a major role because asset investments yield random returns; the valuation of liabilities at market value is also a source of uncertainty.

The goal of the ALM process is to enable payment of current and future pensions. This should be done at minimal funding costs, consisting of contributions by active participants of the fund and the sponsor (e.g., the company backing the fund), and subject to laws and the rules specified by the regulation authority for pension funds. In addition, the outcome has to confirm to the long-term policy rules of the pension fund.

Thus, in general terms, the ALM problem is to select decisions on allocation of the assets, the contributions, indexation of future payments (relative to e.g. wage inflation), etcetera,

W.K. Klein Haneveld · M.H. Streutker · M.H. van der Vlerk ( $\varangle)$

Department of Operations, University of Groningen, PO Box 800, 9700 AV Groningen,

The Netherlands

e-mail: m.h.van.der.vlerk@rug.nl 
which are optimal in some sense, subject to a number of constraints and taking care of uncertainty in an explicit way. All these aspects can be taken care of in a multistage recourse model, which is a model for decision making under uncertainty belonging to the field of stochastic programming. Indeed, multistage recourse models have been applied successfully to a wide range of financial and other problems, see e.g. Ziemba and Mulvey (1998), Ruszczynski and Shapiro (2003), Wallace and Ziemba (2005), and Zenios and Ziemba (2006).

Multistage recourse models comprise additional decisions which allow to react conditionally on new information, becoming available as the future unfolds. The corresponding additional or recourse variables come at certain unit costs, so that the risk associated with a current decision is modeled by assigning additional costs due to uncertainty. Alternatively, one may simply disallow decisions which are too risky (in some well-defined sense). We will use the latter approach to explicitly model short-term risk within a multistage recourse setting.

For the pension fund problem that we study in this paper, long-term solvency goals go together with short-term constraints on the funding ratio, defined as the ratio of assets over liabilities. Due to the uncertainty involved, such a constraint is naturally stated in probabilistic terms. For example, it could be formulated as: The probability that the funding ratio one year from now falls below $105 \%$ should be at most $5 \%$. In stochastic programming terminology, such restrictions on the feasible decisions are called probabilistic or chance constraints. They are closely related to the well-known Value-at-Risk concept used in financial applications.

The inclusion of chance constraints in multistage recourse models for pension fund ALM problems was pioneered by Dert (1995). In this paper we focus on an alternative formulation of short-term risk constraints in ALM models, known as integrated chance constraints. Our motivation to use integrated chance constraints comes both from modeling as well as computational considerations.

The remainder of this paper is organized as follows. First we outline the environment in which the problem is set. In Sect. 2 we then formalize an ALM decision problem for pension funds, arriving at a multistage recourse model. In Sect. 2.2 we motivate and describe in some detail the role and implementation of integrated chance constraints in this model. Relationships to other risk measures are discussed as well. In Sect. 3 we present numerical results for a small example problem.

\subsection{Dutch pension funds and regulation}

Before entering upon these detailed issues, we first present some general background information on Dutch pension funds, since this is the setting of our ALM application. In The Netherlands, as in several other countries, old-age pensions consist of a state allowance complemented by payments out of pension savings. These savings are accumulated during each worker's active career by paying contributions (a fraction of the wages) to a pension fund, both by the employee and the employer. A pension fund may be related to a single company, a branch of industry, or a specific group of professionals.

In 2005, there are about 700 pension funds in The Netherlands. Their total asset value is of the same order of magnitude as the Dutch GDP, see Table 1. While these data suggest that Dutch pension savings are at a relatively high level, the development of the total funding ratio for Dutch pension funds in Table 2 indicates that there is ample reason for concern: following a period of growth, the funding ratio declined rapidly in 1999-2002, due to the downturn of the stock markets and demographical developments on the liabilities side. The subsequent recovery in the following years was caused by the rallying stock markets and 
Table 1 Total asset value of pension funds as a percentage of GDP (in 2005, source: OECD)

\begin{tabular}{lc}
\hline & Total assets/GDP (in \%) \\
\hline France & 5.8 \\
Germany & 3.9 \\
Italy & 2.8 \\
Netherlands & 124.9 \\
Spain & 9.1 \\
UK & 70.1 \\
US & 98.9 \\
\hline
\end{tabular}

Table 2 Total asset value as a percentage of total liabilities value of Dutch pension funds (source: DNB)

\begin{tabular}{ll}
\hline Year & Funding ratio (in \%) \\
\hline 1997 & 138 \\
1998 & 145 \\
1999 & 150 \\
2000 & 141 \\
2001 & 127 \\
2002 & 109 \\
2003 & 114 \\
2004 & 121 \\
2005 & 125 \\
\hline
\end{tabular}

trimming down of pension rights, see Klein Haneveld et al. (2006). However, in 2005 the presupposedly safe funding ratio of $130 \%$ is still only met by around $40 \%$ of the pension funds.

Thus, the situation has changed dramatically since 2000 . The Dutch regulating authority for pension funds (DNB, see http://www.dnb.nl) has reacted by adapting the rules by which pension funds have to operate. The traditional emphasis on long-term risk changes to a more balanced supervision including short-term risk. The technical details have been in progress for quite some time. In our model (Drijver et al. 2003), we propose implementations of the following three conceptual criteria:

(i) Short term: With high reliability, the funding ratio in the next year should be at least at some level specified by the regulating authority.

(ii) Mid term: Seen over a number of years, the funding ratio may fall short occasionally, but if this happens too often or if the shortage is too large (as defined by the regulating authority), some remedial action is required.

(iii) Long term: The solvency of the pension fund should be sufficiently high, from a goingconcern and/or liquidation perspective.

In this paper, we will focus on the representation of the short-term criterion by means of integrated chance constraints.

\section{Integrated chance constraints in multistage recourse ALM for pension funds}

As explained in the introduction, our strategic ALM problem is a dynamic decision problem under uncertainty. We are asked to come up with decisions such as the contribution rate and 
the portfolio mix, and possibly a remedial action in case the funding ratio is insufficient. These decisions need to be taken right now, in the face of uncertainty about investment yields and other problem parameters.

We consider a large Dutch company which has its own pension fund. It will be assumed that the pension plan is defined benefit, and that the company acts as sponsor of the fund: if the funding ratio is too low, the company may be obliged to provide (or guarantee) additional money. Mulvey et al. (2003, 2008) describe a multi-period network model where the financial health of a company is optimized by integrating the corporate financial and pension planning. However, in the Netherlands, pension funds are independent institutions by law, making it impossible to integrate both plans.

Thus, the pension fund has three sources of funding its liabilities: revenues from its asset portfolio, regular contributions made by the company and the participants of the fund, and remedial contributions made by the company. The pension fund has to decide periodically how to distribute the investments over different asset classes and what the contribution rate should be in order to meet all its obligations.

Within a pension fund, there are typically different groups of participants, who all have their own interests. For example, active participants (workers) prefer to pay a low contribution rate, whereas passive participants (retirees) are concerned about the level of current payments (indexation). The company prefers low contribution rates, and wishes to avoid remedial contributions. The ALM model should adequately reflect the-often conflictingobjectives of all interest groups. This is achieved by means of constraints and/or penalty terms in the objective function.

In addition, as already mentioned in the Introduction, there are a number of regulatory rules which further restrict the decisions of the pension fund. The long-term solvency requirements are reflected by the multi-stage character of the model. For the inclusion of the mid-term requirements, which involves binary decision variables, we refer to the thesis of Drijver (2005). Foremost, the introduction of binary variables in the model is justified by the fact that they are necessary to include several realistic features in our multistage recourse ALM model. In addition, we have a long-standing interest in (computational) properties of recourse models with integer variables, see e.g. Louveaux and Van der Vlerk (1993), Klein Haneveld and Van der Vlerk (1999), Van der Vlerk (2004). How to model the short-term criterion is discussed in detail in Sect. 2.2.

In the following we will look at a multi-stage recourse model representing this problem. We will focus on the asset side of the problem, so the indexation policies are left out of consideration.

\subsection{Setting}

We split the planning horizon in $T$ subperiods of one year each, and denote the resulting time stages by an index $t$. Time $t=0$ is the current time and $t=T$ is the length of the horizon. Year $t(t=1, \ldots, T)$ is the span of time $[t-1, t)$.

The uncertainty is modeled through a large but finite number $S$ of scenarios. Each scenario represents a possible realization of all uncertain parameters in the model. Let $\omega_{t}$ represent the vector of random parameters whose values are revealed in year $t$. Then the set of all scenarios is the set of all realizations $\left(\omega_{1}^{s}, \ldots, \omega_{T}^{s}\right), s \in \mathcal{S}:=\{1, \ldots, S\}$, of $\left(\omega_{1}, \ldots, \omega_{T}\right)$. Each scenario $s$ has a probability $p^{s}$, where $p^{s}>0$ and $\sum_{s=1}^{S} p^{s}=1$. Since in a dynamic model information on the actual values of the uncertain parameters is revealed in stages, a suitable representation of the set of scenarios is given by a scenario tree. An example of a scenario tree is given in Fig. 1. Each path from $t=0$ to $t=T$ represents one scenario. 


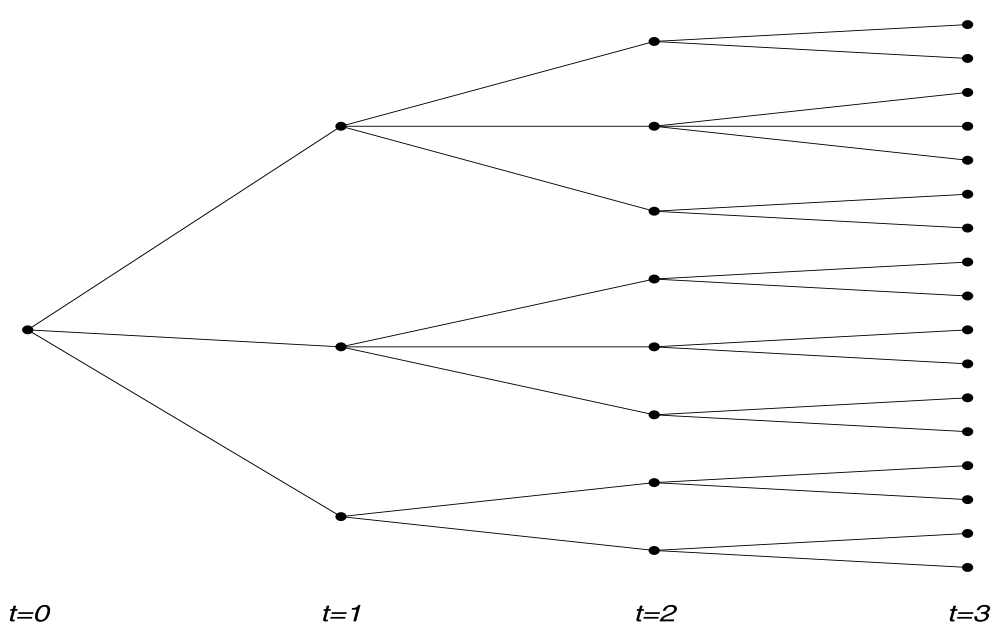

Fig. 1 Example of a scenario tree, with $T=3$ and $S=17$

At every node $(t, s)$ of the tree, decisions are to be made which are optimal given the history up to then and under uncertainty about the remaining future. In the corresponding scenario representation of the problem, explicit constraints are added to enforce nonanticipativity of the decisions.

\subsection{Integrated chance constraints}

The short-term criterion proposed in Sect. 1.1 reads that next years funding ratio should be no lower than a given level $\alpha$, say $\alpha=105 \%$, with high reliability. The formulation of this criterion clearly indicates its stochastic nature, which should be reflected in the way it is modeled. Starting from traditional chance constraints, we will arrive at our implementation of an adapted short-term criterion by means of integrated chance constraints.

\subsubsection{Conceptual motivation}

In our ALM model, a direct translation of the short-term criterion would be the chance constraint

$$
\operatorname{Pr}\left\{F_{t+1}^{*} \geq \alpha \mid(t, s)\right\} \geq \gamma_{t}
$$

where $F_{t+1}^{*}$ is the funding ratio just before a possible remedial contribution by the sponsor, $\gamma_{t}$ is the required reliability at time $t$ (e.g. $\gamma_{t}=0.95$ ), and the notation indicates that probability is measured conditional on $(t, s)$ being the current node. Using that the funding ratio is defined as the ratio of the assets $A_{t+1}^{*}$ over liabilities $L_{t+1}$, an equivalent formulation is

$$
\operatorname{Pr}\left\{A_{t+1}^{*}-\alpha L_{t+1} \geq 0 \mid(t, s)\right\} \geq \gamma_{t} .
$$

Note that the risk measure underlying such a chance constraint is qualitative, since it measures the probability of a shortfall of the funding ratio, but the magnitude of the shortage is not taken into account. In other applications this may be justified or even preferable, but in an ALM model the size of the funding shortage is obviously relevant. 
We propose to replace the current qualitative criterion by a quantitative one-year risk measure. In our ALM model, this role is played by integrated chance constraints. To introduce this concept, we return to the chance constraint, and will see that it comes up in a natural way when we look at computational issues.

\subsubsection{Computational motivation and definition}

In the chance constraint (1), both $A_{t+1}^{*}$ and $L_{t+1}$ are random quantities because they depend on underlying random parameters such as asset yields. Moreover, they depend linearly on the current decisions $x_{t}^{s}$ which involve asset allocation and contributions. To stress these relations and in order to simplify the notation, for the time being we will use the generic representation of an (individual) chance constraint

$$
\operatorname{Pr}\{B x-d \geq 0\} \geq \gamma,
$$

where $x$ is an $n$-vector of decision variables and the $n$-vector $B$ and the scalar $d$ are both random parameters. Assume that $(B, d)$ follow a finite discrete distribution with realizations $\left(B^{s}, d^{s}\right)$ and corresponding probabilities $p^{s}, s \in \mathcal{S}:=\{1, \ldots, S\}$.

All other constraints in our ALM model are linear, and also the chance constraint can be represented by linear constraints, as follows.

$$
\begin{aligned}
& B^{s} x+\delta^{s} M \geq d^{s}, \quad s \in \mathcal{S}, \\
& \sum_{s \in \mathcal{S}} p^{s} \delta^{s} \leq 1-\gamma, \\
& \delta^{s} \in\{0,1\}, \quad s \in \mathcal{S},
\end{aligned}
$$

where $M$ is a sufficiently large number. Note that this formulation necessarily uses binary variables $\delta^{s}, s \in \mathcal{S}$, to indicate realizations $\left(B^{s}, d^{s}\right)$ which are unfavorable for $x$, e.g., which would result in underfunding in the ALM model. The probability weighted average of these binary variables then equals the risk of underfunding associated with the decision $x$, which should be at most $1-\gamma$. Because of these binary variables, the inclusion of a chance constraint at every node $(t, s)$ (except at the end nodes) of our multistage recourse model would have severe consequences for the computational tractability of the model.

For problems involving binary (or general integer) decision variables, a natural approach is to relax the integrality restrictions and solve the resulting relaxation. In our case, such a relaxation transforms the mixed-integer linear representation of the chance constraint into a system of linear constraints in continuous variables, which is equivalent to

$$
\begin{aligned}
& B^{s} x+y^{s} \geq d^{s}, \quad s \in \mathcal{S}, \\
& \sum_{s \in \mathcal{S}} p^{s} y^{s} \leq \beta, \\
& y^{s} \geq 0, \quad s \in \mathcal{S},
\end{aligned}
$$

where the parameter $\beta$ is non-negative. By the first set of inequalities, for each $s$ the nonnegative variable $y^{s}$ is not less than the shortfall $\left(B^{s} x-d^{s}\right)^{-}$, where $(a)^{-}:=\max \{-a, 0\}$ is the negative part of $a \in \mathbb{R}$. The next inequality therefore puts an upper bound $\beta$ on the expected shortfall. That is, the system (2) is equivalent to

$$
\sum_{s \in \mathcal{S}} p^{s}\left(B^{s} x-d^{s}\right)^{-} \leq \beta,
$$


or

$$
\mathbb{E}\left[(B x-d)^{-}\right] \leq \beta
$$

with $\mathbb{E}$ denoting expectation with respect to the distribution of $(B, d)$. Such constraints, bounding an expected shortfall, were named integrated chance constraints by Klein Haneveld (1986), since it can be shown that

$$
\mathbb{E}\left[(B x-d)^{-}\right]=\int_{-\infty}^{0} \operatorname{Pr}\{B x-d<u\} d u .
$$

The integrand in (4) is the complement of the probability $\operatorname{Pr}\{B x-d \geq u\}$; it appears in an equivalent risk version

$$
\operatorname{Pr}\{B x-d<u\} \leq 1-\gamma(u)
$$

of the underlying chance constraint, where the parameter $1-\gamma(u)$ denotes the maximum acceptable risk of not meeting the target level $u$.

Loosely speaking, the identity (4) shows that an integrated chance constraint corresponds to some aggregation of the infinitely many chance constraints which-in theory-could be defined for all possible target levels $u \leq 0$. By indicating, through the corresponding risk parameters $1-\gamma(u)$, that larger shortages are even less acceptable than smaller ones, this indeed results in a quantitative risk measure defined in terms of traditional, qualitative chance constraints.

\subsubsection{Discussion}

From optimization and calculation point of view, chance constraints have disadvantages, since they may give rise to nonconvexity, see Klein Haneveld (1986), leading to the need of binary decision variables. Integrated chance constraints, based on a quantitative risk measure, do not have these disadvantages, and are tractable computationally, see Klein Haneveld and Van der Vlerk (2006).

The use of chance constraints corresponds to Value-at-Risk (VaR)-based risk management in finance. VaR is very popular in practice, but critical comments are relevant, too. Basak and Shapiro (2001) use financial economic theory to show that the use of VaR exhibits problematic features. Moreover, these shortcomings are remedied when the agents in the equilibrium model are supposed to apply a quantitative risk concept, called limitedexpected-losses (LEL). This approach corresponds to the integrated chance constraint concept.

A second improvement of the VaR approach is provided by the concept of Conditional VaR (CVaR), see e.g. Uryasev (2000), Rockafellar and Uryasev (2000), and Topaloglou et al. (2002). This risk concept is shown to be coherent (Pflug 2000 and Rockafellar and Uryasev 2002). As a matter of fact, our integrated chance constraints are closely related to constraints on a variant of CVaR, the Conditional Surplus-at-Risk (CSaR), see Klein Haneveld and Van der Vlerk (2006). Both modeling concepts use a quantitative risk measure to be controlled. But the difference is, that in an integrated chance constraint the shortage is measured with respect to some a priori chosen threshold parameter, whereas in a CSaR constraint the threshold is equal to the surplus-at-risk, which itself is an outcome of the optimization process. So, both integrated chance constraints and CSaR avoid the problems coming from qualitative risk measures. But the former are easier to implement in calculations. 


\subsubsection{Implementation as short-term risk constraints}

We conclude that integrated chance constraints provide a suitable way to model short-term risk in an ALM model, both from conceptual as well as computational point of view. Returning to the specific notation of our ALM model, we thus include an integrated chance constraint

$$
\mathbb{E}\left[\left(A_{t+1}^{*}-\alpha L_{t+1}\right)^{-} \mid(t, s)\right] \leq \beta_{t}
$$

in every subproblem $(t, s), t<T$, of our multistage recourse model. They reflect our alternative short-term criterion, stating that next years funding ratio should be such that the expected funding shortfall is at most $\beta_{t}$, given that the current state is $(t, s)$.

The parameters $\beta_{t}, t=0, \ldots, T-1$, giving the maximal acceptable expected funding shortage, of course need to be specified numerically. In general it is harder to come up with these values than it would be for the reliability parameters $\alpha_{t}$, as required for traditional chance constraints. The latter parameters are scale free, and correspond to a risk notion which is more familiar to e.g. managers of pension funds.

With $S_{t}^{s}$ denoting the number of possible realizations from $(t, s)$ in year $t+1$, it follows from (2) that inclusion of the integrated chance constraint (5) in subproblem $(t, s)$ comes at the price of $S_{t}^{s}$ additional continuous variables and $S_{t}^{s}+1$ additional linear constraints. In many applications, e.g. in the numerical example below, $S_{t}^{s}$ is in the order of 5 to 10 , so that this extension of the model provides no computational hardship.

In case the number of realizations is substantially larger, say 1000 or more, the linear programming (LP) representation (2) becomes inefficient. In Klein Haneveld and Van der Vlerk (2006) it is shown that the induced feasible set $C(\beta)$, corresponding to an integrated chance constraint (3) with an underlying finite discrete distribution on $S$ points, is given by

$$
C(\beta)=\bigcap_{K \subset \mathcal{S}}\left\{x \in \mathbb{R}^{n}: \sum_{k \in K} p^{k}\left(d^{k}-B^{k} x\right) \leq \beta\right\} .
$$

Since there are $2^{S}-1$ non-empty subsets of $\mathcal{S}=\{1, \ldots, S\}$, it follows that $C(\beta)$ is a polyhedral set defined by as many linear constraints. For any non-trivial number of realizations $S$, it is obviously not sensible (if at all possible) to explicitly include all of them in the model. However, the representation (6) underlies a very efficient cutting plane algorithm for solving LP problems with (variants of) integrated chance constraints. For a small example problem with 1000 realizations, an optimal solution is found after generating only 9 out of the approximately $10^{300}$ constraints defining the set $C(\beta)$. Further numerical evidence shows that the cutting plane algorithm is much faster than the straightforward LP approach on larger problem instances.

\section{Numerical illustration}

In this section we present numerical results for a small instance of a multistage ALM model. The main purpose is to illustrate the effect of including short-term risk constraints, modeled as integrated chance constraints. In this example we include such a constraint only at $t=0$,

$$
\mathbb{E}\left[\left(A_{1}^{*}-\alpha L_{1}\right)^{-}\right] \leq \beta,
$$

thus requiring current decisions which are not too risky, measured by the expected shortfall below the target funding level at $t=1$. 


\subsection{Model description}

We define the following indices, variables, random parameters and deterministic parameters. (To simplify the notation, we omit the scenario index $s$.)

Indices

$t$ time index, $t=0,1, \ldots, T$

$i \quad$ index of asset classes, $i=1, \ldots, N$

\section{Variables}

$Z_{t} \quad$ remedial contribution by the sponsor at time $t$

$X_{i t} \quad$ value of investments in asset class $i$, at the beginning of year $t$

$c_{t+1} \quad$ contribution rate for year $t+1$

$A_{t} \quad$ total asset value at time $t$

$A_{t}^{*} \quad$ total asset value at time $t$ just before a possible remedial contribution $Z_{t}$

$\Delta_{i t}^{+} \quad$ value of assets in class $i$ bought at time $t$

$\Delta_{i t}^{-} \quad$ value of assets in class $i$ sold at time $t$

$U_{T} \quad$ underfunding at time horizon with respect to the original funding ratio $A_{0} / L_{0}$

\section{Random parameters}

$r_{i t} \quad$ random return on asset class $i$ in year $t$

$W_{t}$ random total wages of active participants in year $t$

$P_{t} \quad$ random total benefit payments in year $t$

$L_{t} \quad$ random value of liabilities after year $t$

\section{Deterministic parameters}

$k_{i} \quad$ proportional transaction cost for asset class $i$

$w_{i}^{l} \quad$ lower bound on the value of asset class $i$ as a fraction of the total asset portfolio

$w_{i}^{u} \quad$ upper bound on the value of asset class $i$ as a fraction of the total asset portfolio

$c^{l} \quad$ lower bound on the contribution rate

$c^{u} \quad$ upper bound on the contribution rate

$\alpha \quad$ lower bound on the funding ratio

$\gamma_{t} \quad$ discount factor for a cash flow in year $t$

$\lambda_{Z}$ penalty parameter for a remedial contribution

$\lambda_{U}$ penalty parameter for underfunding at time horizon

The decisions at time $t \in \mathcal{T}_{0}:=\{0,1, \ldots, T-1\}$ are denoted by the vector

$$
x_{t}=\left(Z_{t}, X_{1 t}, \ldots, X_{N t}, c_{t+1}\right) \text {. }
$$

At the time horizon $t=T$, only the decision $Z_{T}$ occurs. The variables $A_{t}, \Delta_{1 t}^{+}, \ldots, \Delta_{N t}^{+}$, $\Delta_{1 t}^{-}, \ldots, \Delta_{N t}^{-}$, and $U_{T}$ are state variables. They are determined by the parameters and the decision variables.

The state variable $A_{t}^{*}$, denoting the value of the assets just before a possible remedial payment $Z_{t}$, is used to model the short-term risk criterion, and to determine the size of the remedial contribution (if any): it must hold that $Z_{t} \geq\left(A_{t}^{*}-\alpha L_{t}\right)^{-}$.

The randomness of the asset returns and the total wages of active participants is obvious. The randomness of the liabilities results from their valuation at market value and inflation developments. The latter causes the randomness in the benefit payments as well. For $t \in$ 
$\mathcal{T}_{1}:=\{1,2, \ldots, T\}$, we define the random vector $\omega_{t}$ with realizations

$$
\omega_{t}^{s}=\left(r_{1 t}^{s}, \ldots, r_{N t}^{s}, W_{t}^{s}, P_{t}^{s}, L_{t}^{s}\right), \quad s \in \mathcal{S} .
$$

The constraints in the model can be divided into two types of constraints: actuarial principles, and policies. In each scenario $s$ and at every time $t$ (that is, in each node $(t, s)$ ), the constraints must be satisfied. As before, we omit the index $s$ in our presentation of the constraints below.

Let us first formulate the constraints based on actuarial principles:

$$
A_{t}=\sum_{i=1}^{N}\left(1+r_{i t}\right) X_{i t}+c_{t} W_{t}-P_{t}+Z_{t}, \quad t \in \mathcal{T}_{1}
$$

The total value of the assets at time $t$. Note that $A_{t}=A_{t}^{*}+Z_{t}$.

$$
X_{i, t+1}=\left(1+r_{i t}\right) X_{i t}-\Delta_{i t}^{-}+\Delta_{i t}^{+}-k_{i}\left(\Delta_{i t}^{-}+\Delta_{i t}^{+}\right), \quad i=1, \ldots, N, t \in \mathcal{T}_{0}
$$

The value of the investments in asset class $i$, at the beginning of the year $t+1$.

$$
\sum_{i=1}^{N}\left(X_{i, t+1}+k_{i}\left(\Delta_{i t}^{-}+\Delta_{i t}^{+}\right)\right)=A_{t}, \quad t \in \mathcal{T}_{0}
$$

All assets should be allocated.

The policies of the pension fund are reflected by the following constraints:

$w_{i}^{l} \sum_{j=1}^{N} X_{j t} \leq X_{i t} \leq w_{i}^{u} \sum_{j=1}^{N} X_{j t}, \quad i=1, \ldots, N, t \in \mathcal{T}_{1}$

Lower and upper bounds on the value of asset class $i$ as a fraction of the total asset portfolio.

$c^{l} \leq c_{t} \leq c^{u}, \quad t \in \mathcal{T}_{1}$

Lower and upper bounds on the contribution rate.

$A_{t} \geq \alpha L_{t}, \quad t \in \mathcal{T}_{1}$

Lower bound on the funding ratio $F_{t}:=A_{t} / L_{t}$. The level $\alpha$ is prescribed by the regulator.

$$
A_{T}+U_{T} \geq F_{0} L_{T}
$$

It is desired that the funding ratio at the time horizon is not below the funding ratio at $t=0$.

Finally, the integrated chance constraint $\mathbb{E}\left[\left(A_{1}^{*}-\alpha L_{1}\right)^{-}\right] \leq \beta$, reflecting the short-term risk criterion, is implemented as a system of linear constraints analogous to (2).

The pension fund aims to minimize the total expected cost of funding, i.e., the contribution rates for the active participants and the company, and the remedial contributions. Moreover, penalty costs are assigned to the undesirable events: remedial contributions, and a low funding ratio at the planning horizon. All these components together constitute the objective function:

$$
\mathbb{E}\left[\sum_{t=0}^{T} \gamma_{t}\left(c_{t} W_{t}+\lambda_{Z} Z_{t}\right)+\gamma_{T} \lambda_{U} U_{T}\right] .
$$

\subsection{Data}

In this example model we consider four asset classes $(N=4)$, whose deterministic properties are described in Table 3. All amounts are in millions of euros.

The values of the other deterministic parameters and parameters observed at $t=0$ are shown in Table 4. 
Table 3 Data on the asset classes

Table 4 Values of some deterministic parameters, and observed random parameters and contribution rate at $t=0$

\begin{tabular}{llllll}
\hline Asset class & $i$ & $w_{i}^{l}$ & $w_{i}^{u}$ & $k_{i}$ & Initial investments \\
\hline Stocks & 1 & 0.30 & 0.60 & 0.00425 & 7656 \\
Bonds & 2 & 0.30 & 0.60 & 0.00150 & 4048 \\
Real estate & 3 & 0.10 & 0.25 & 0.00425 & 3080 \\
Cash & 4 & 0 & 0.20 & 0.00050 & 1056 \\
\hline
\end{tabular}

\begin{tabular}{lll}
\hline$c^{l}=0$ & $\gamma_{0}=1$ & $W_{0}=10520$ \\
$c^{u}=0.21$ & $\gamma_{1}=0.9693$ & $P_{0}=1115$ \\
$\alpha=1.05$ & $\gamma_{2}=0.9308$ & $L_{0}=15200$ \\
$\lambda_{U}=1.20$ & $\gamma_{3}=0.8873$ & $c_{0}=0.17$ \\
$\lambda_{Z}=1.25$ & & \\
\hline
\end{tabular}

Table 5 Summary statistics for the random returns in year 1

\begin{tabular}{lllrrrrr}
\hline Parameter & Mean & $\begin{array}{l}\text { Standard } \\
\text { deviation }\end{array}$ & \multicolumn{6}{l}{ Correlations } \\
\cline { 5 - 8 } & & $r_{11}$ & \multicolumn{1}{l}{$r_{12}$} & \multicolumn{1}{c}{$r_{13}$} & \multicolumn{1}{c}{$r_{14}$} \\
\hline$r_{11}$ & 0.068 & 0.159 & 1.00 & 0.50 & -0.11 & 0.13 \\
$r_{12}$ & 0.058 & 0.060 & 0.50 & 1.00 & 0.17 & -0.22 \\
$r_{13}$ & 0.065 & 0.112 & -0.11 & 0.17 & 1.00 & -0.31 \\
$r_{14}$ & 0.032 & 0.017 & 0.13 & -0.22 & -0.31 & 1.00 \\
\hline
\end{tabular}

The model has 4 stages, allowing for decisions at $t=0$ (now) up to the time horizon $T=3$. For each year $[t-1, t), t=1,2,3$, the asset returns, wages, benefit payments, and liabilities are modeled by random parameters. For future reference, some statistics on the distribution of the asset returns for $t=1$ are presented in Table 5 .

For further details on the distribution and the construction of the scenario tree for this example, we refer to the $\mathrm{PhD}$ thesis of Drijver (2005).

In each state of the world before the horizon, uncertainty about the future is represented by 10 different realizations of the random parameters in each of the remaining years. Thus, there are $S=10^{T}=1000$ scenarios in total. In each node, the conditional probabilities are 0.1 , so that each scenario $s$ has probability $p^{s}=0.001$.

Using the data of the Tables 3 and 4, we compute that the current asset value $A_{0}$ equals 16513.4 million euro. Thus, the initial funding ratio $F_{0}=A_{0} / L_{0}$ equals 1.09 , which is above the threshold value $\alpha=1.05$.

\subsection{Results}

All numerical results were obtained using the model management system SLP-IOR (see e.g. Kall and Mayer 1996, 2004, 2005). Because of the small size of the recourse model, the equivalent LP model could be solved by any of the LP codes available in SLP-IOR in negligible time.

Initially, we solved the ALM model without an integrated chance constraint at $t=0$. It appears that for the optimal solution, the expected shortfall of the assets with respect to $\alpha$ times the liabilities at $t=1$ equals 647 million euro ( $3.9 \%$ of $A_{0}$ ). Subsequently, we solved the model including the integrated chance constraint, restricting the expected shortfall at 
Table 6 Results for different values of $\beta$

\begin{tabular}{llllllllllr}
\hline$\beta$ & Opt. val. & Direct & Future & $X_{10}$ & $X_{20}$ & $X_{30}$ & $X_{40}$ & $c_{1}$ & $Z_{0}$ \\
\hline$\infty$ & 4686 & 1318 & 3368 & 7427 & 4951 & 4126 & 0 & 0.128 & 0 \\
600 & 4687 & 1418 & 3268 & 7427 & 4951 & 4126 & 0 & 0.138 & 0 \\
550 & 4688 & 1540 & 3148 & 7427 & 4951 & 4126 & 0 & 0.150 & 0 \\
500 & 4690 & 1661 & 3029 & 7427 & 4951 & 4126 & 0 & 0.161 & 0 \\
450 & 4694 & 1782 & 2911 & 7427 & 4951 & 4126 & 0 & 0.173 & 0 \\
400 & 4703 & 1945 & 2757 & 7158 & 5220 & 4126 & 0 & 0.199 & 0 \\
350 & 4720 & 2107 & 2612 & 6887 & 5489 & 4125 & 0 & 0.205 & 0 \\
300 & 4767 & 2268 & 2498 & 6949 & 6465 & 3183 & 0 & 0.210 & 92 \\
250 & 4829 & 2469 & 2359 & 6660 & 7016 & 3080 & 0 & 0.210 & 252 \\
200 & 4896 & 2674 & 2222 & 6078 & 7447 & 3080 & 310 & 0.210 & 416 \\
150 & 4998 & 2937 & 2060 & 5477 & 6713 & 3080 & 1855 & 0.210 & 626 \\
100 & 5108 & 3171 & 1936 & 5974 & 5661 & 2335 & 3342 & 0.210 & 814 \\
50 & 5233 & 3472 & 1761 & 5264 & 6561 & 2213 & 3509 & 0.210 & 1055 \\
0 & 5529 & 4147 & 1381 & 5428 & 5967 & 3080 & 3619 & 0.210 & 1595 \\
\hline
\end{tabular}

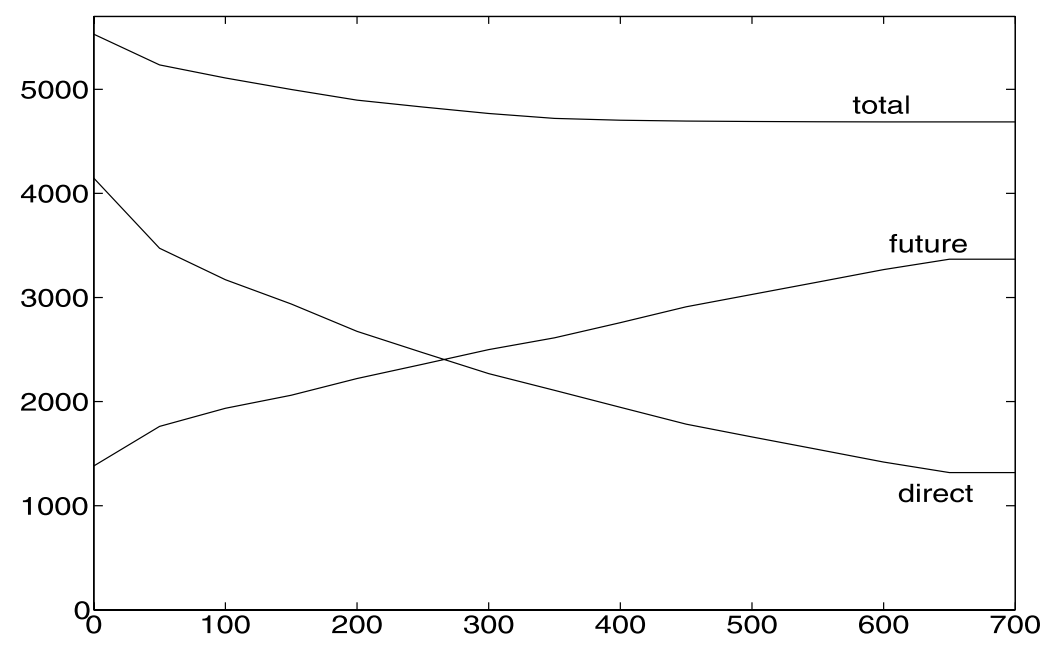

Fig. 2 Optimal value (direct and expected future costs) as function of $\beta$

$t=1$ by several values of the right-hand side parameter $\beta$. The resulting optimal values and first-stage solutions are presented in Table 6.

Of course, the optimal value increases with decreasing values of $\beta$. However, as illustrated in Fig. 2, the increase is relatively small (less than 2\%) for values of $\beta$ as small as 300 ( $1.8 \%$ of $\left.A_{0}\right)$. The influence on the composition of the total costs is much stronger: over this range, direct costs increase sharply $(72 \%)$, whereas expected future costs fall $(26 \%)$.

Next we consider the effect of the integrated chance constraint on the first-stage decisions, i.e., on the contribution rate, the asset mix, and the remedial contribution at $t=0$. For $\beta$ decreasing to 450 , we see that the request for higher short-term reliability is satisfied by increasing the contribution rate. But then, as $\beta$ keeps decreasing, also the asset mix changes 


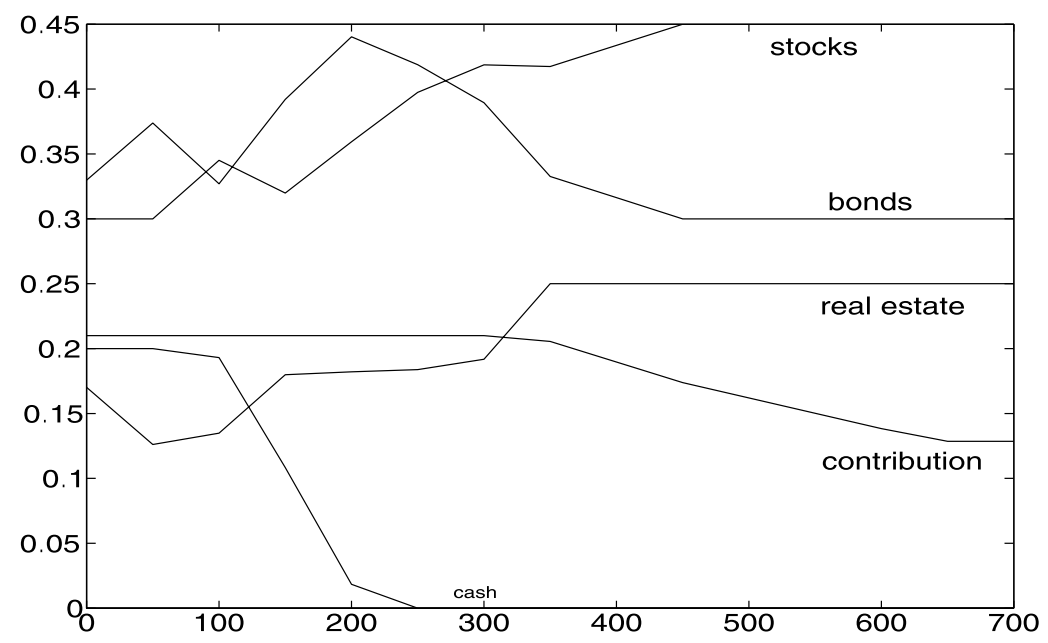

Fig. 3 Asset mix and contribution rate as function of $\beta$

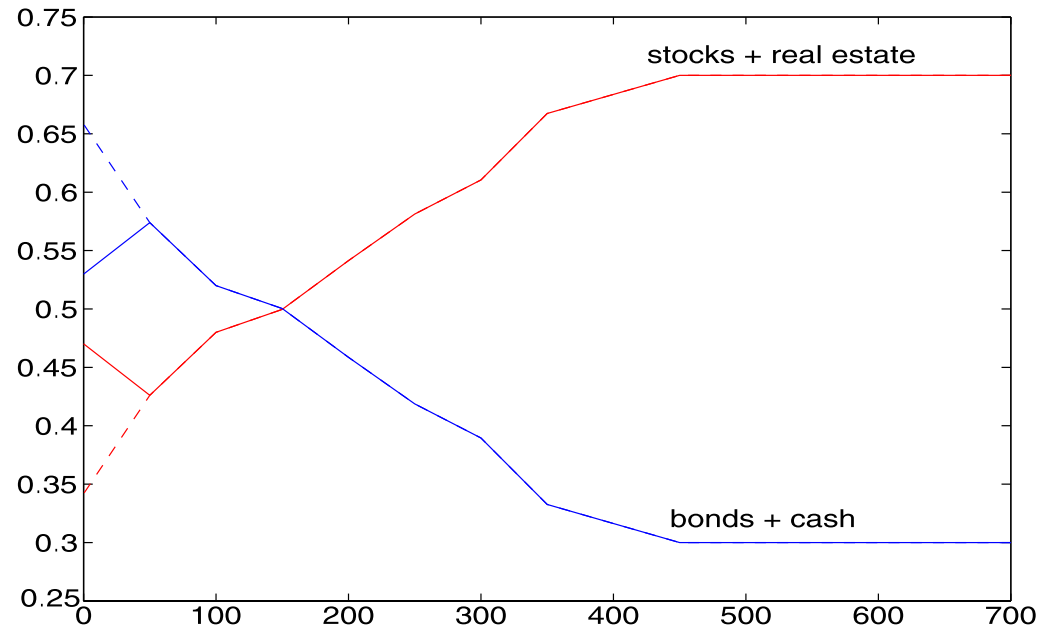

Fig. 4 Aggregated asset mix as function of $\beta$ (dashed: relaxed bounds)

gradually: less is invested in relatively risky stocks and real estate, and more in bonds and eventually also cash. For lower values of $\beta$ this pattern is less clear when observing the individual asset classes as depicted in Fig. 3. On the aggregate level, considering the risky assets versus bonds and cash, the pattern is clearly shown in Fig. 4 (solid lines) except for $\beta=0$. The latter is caused by binding lower and upper bounds on the relative amounts of stocks and cash, respectively, as is illustrated by the dashed graphs in Fig. 4 depicting the aggregated asset mix which results when these bounds are relaxed.

For $\beta$ lower than 350, remedial contributions $Z_{0}$ are part of the optimal solution at $t=0$, see Table 6 and Fig. 5. On first sight, they appear to be larger than necessary. For example, to accommodate a decrease of $\beta$ from 300 to 250 , one could reason that an increase of $Z_{0}$ with 50 should be sufficient. However, due to the penalty parameter $\lambda_{Z}=1.25$, this would 


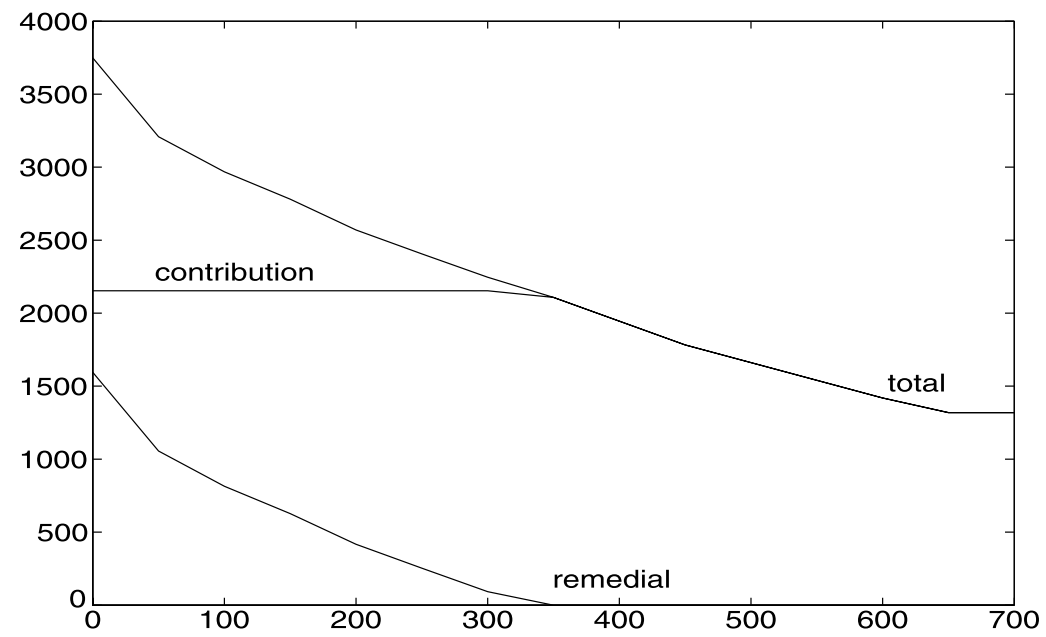

Fig. 5 Funding costs at $t=0$ as function of $\beta$

increase direct costs by 62.5. In the presented optimal solution, in which $Z_{0}$ is increased by 160 at additional direct costs of 200, this is (more than) compensated by the resulting decrease of the expected future costs, since the additional assets allow for lower funding costs in later stages. Obviously, other choices for the parameter $\lambda_{Z}$ will result in a different balance between direct and expected future costs.

We conclude that, at least in this example problem, the integrated chance constraint on the expected funding shortage appears to be a suitable tool for modeling short-term risk. In particular, for appropriately chosen values of the parameter $\beta$, it may lead to a solution which has lower short-term risk in exchange for marginally higher expected total costs. It should be stressed, however, that selecting a good value for $\beta$ a priori can be difficult in practice. Given the low computational costs of including integrated chance constraints, it may be feasible to find a reasonable parameter setting by numerical experiments.

Finally, let us consider the reliability of the optimal solutions in terms of the probability $\operatorname{Pr}\left\{A_{1}^{*}-\alpha L_{1} \geq 0\right\}$, i.e., the reliability concept underlying traditional chance constraints. The solution of the model without integrated chance constraint turns out to have reliability 0.5 , whereas e.g. the solution of the integrated chance constraint model with $\beta=450$ is reliable at the 0.7 level, and $\beta=200$ yields reliability 0.8 . In this example, it appears that the reliability of the solutions increases (step-wise) with decreasing $\beta$. In general, however, this need not be the case. For example, a solution which yields small shortages for every realization is feasible with respect to the integrated chance constraint for some small $\beta$, whereas it has reliability 0 .

In chance-constrained models, the reliability is usually required to be at least 0.9 , which is obtained in this model by setting $\beta=72$. For the current model, higher aspiration levels actually imply $100 \%$ reliability (corresponding to the extreme setting $\beta=0$ ), due to the fact that each realization at $t=1$ has probability 0.1 . Indeed, this is a general modeling weakness of chance constraints based on a small number of possible realizations, as is often the case for single-period risk constraints in multi-stage recourse models of realistic size. 


\section{Summary and concluding remarks}

We motivated and described the role played by integrated chance constraints in an ALM model for Dutch pension funds. To set the stage, we outlined the practical setting as well as our modeling approach for this dynamic decision problem under uncertainty.

Integrated chance constraints are appropriate for modeling single-period risk constraints, in particular if a quantitative risk measure is preferable, as is the case here. Moreover, they are computationally attractive in the given multistage recourse setting, since they can be formulated in terms of a limited number of linear constraints without the need to introduce additional binary decision variables.

These claims are supported by the numerical results on a small example problem. No computational results on (semi-)realistic data are available at this time. However, such data have been made available to us by a major Dutch pension fund. Initial outcomes of an ALM model including integrated chance constraints and implementing the mid-term risk criterion as described in the previous section, obtained with a special purpose heuristic, are reported in the $\mathrm{PhD}$ thesis of Drijver (2005), and in other publications.

We expect that such multistage recourse models, including implementations of various risk criteria, will prove to be a useful tool in strategic ALM studies for pension funds. Even though we believe that this approach allows to model important aspects in a realistic way, the final judgment on such models will have to come from the analysis of numerical results for (semi-)realistic problems.

Open Access This article is distributed under the terms of the Creative Commons Attribution Noncommercial License which permits any noncommercial use, distribution, and reproduction in any medium, provided the original author(s) and source are credited.

\section{References}

Basak, S., \& Shapiro, A. (2001). Value-at-Risk-based risk management: Optimal policies and asset prices. The Review of Financial Studies, 14(2), 371-405.

Dert, C. L. (1995). Asset liability management for pension funds, a multistage chance constrained programming approach. $\mathrm{PhD}$ thesis, Erasmus University, Rotterdam, The Netherlands.

Drijver, S. J. (2005). Asset liability management for pension funds using multistage mixed-integer stochastic programming. $\mathrm{PhD}$ thesis, University of Groningen.

Drijver, S. J., Klein Haneveld, W. K., \& van der Vlerk, M. H. (2003). Asset liability management modeling using multistage mixed-integer stochastic programming. In B. Scherer (Ed.), Asset and liability management tools: A handbook for best practice (pp. 309-324). London: Risk Books. Chapter 16.

Kall, P., \& Mayer, J. (1996). SLP-IOR: An interactive model management system for stochastic linear programs. Mathematical Programming, 75(2), 221-240.

Kall, P., \& Mayer, J. (2004). Modeling support for multistage recourse problems. In Lecture notes in econom. and math. systems: Vol. 532. Dynamic stochastic optimization (Laxenburg, 2002) (pp. 21-41). Berlin: Springer.

Kall, P., \& Mayer, J. (2005). Building and solving stochastic linear programming models with SLP-IOR. In S. W. Wallace \& W. T. Ziemba (Eds.), MPS-SIAM series in optimization. Applications of stochastic programming (pp. 79-93). Philadelphia: SIAM. Chapter 6.

Klein Haneveld, W. K. (1986). In Lecture notes in economics and mathematical systems: Vol. 274. Duality in stochastic linear and dynamic programming. Berlin: Springer.

Klein Haneveld, W. K., \& van der Vlerk, M. H. (1999). Stochastic integer programming: General models and algorithms. Annals of Operations Research, 85, 39-57.

Klein Haneveld, W. K., \& van der Vlerk, M. H. (2006). Integrated chance constraints: reduced forms and an algorithm. Computational Management Science, 3(4), 245-269.

Klein Haneveld, W. K., Streutker, M. H., \& van der Vlerk, M. H. (2006). Modeling ALM for Dutch pension funds. In J. Safrankova \& J. Pavlu (Eds.), WDS'06 proceedings of contributed papers: Part Imathematics and computer sciences (pp. 100-105). Prague: Matfyzpress. 
Louveaux, F. V., \& van der Vlerk, M. H. (1993). Stochastic programming with simple integer recourse. Mathematical Programming, 61, 301-325.

Mulvey, J. M., Pauling, B., \& Simsek, K. D. (2003). A stochastic network approach for integrating pension and corporate financial planning. In A. Nagurney (Ed.), Innovations in financial and economic networks (pp. 67-83). Cheltenham: Edward Elgar. Chapter 4.

Mulvey, J. M., Simsek, K. D., Zhang, Z., Fabozzi, F. J., \& Pauling, B. (2008). Assisting defined-benefit pension plans. Operations Research, 56(5), 1066-1078.

Pflug, G. Ch. (2000). Some remarks on the value-at-risk and the conditional value-at-risk. In S. P. Uryasev (Ed.), Nonconvex optimization and its applications: Vol. 49. Probabilistic constrained optimization (pp. 272-281). Dordrecht: Kluwer Academic

Rockafellar, R. T., \& Uryasev, S. (2000). Optimization of conditional value-at-risk. The Journal of Risk, 2(3), 21-41.

Rockafellar, R. T., \& Uryasev, S. (2002). Conditional value-at-risk for general loss distributions. Journal of Banking and Finance, 26(7), 1443-1471.

Ruszczynski, A., \& Shapiro, A. (Eds.) (2003). Handbooks in operations research and management science: Vol. 10. Stochastic programming. Amsterdam: Elsevier.

Topaloglou, N., Vladimirou, H., \& Zenios, S. A. (2002). CVaR models with selective hedging for international asset allocation. Journal of Banking and Finance, 26(7), 1535-1561.

Uryasev, S. P. (Ed.) (2000). Probabilistic constrained optimization. Dordrecht: Kluwer Academic. Methodology and applications.

van der Vlerk, M. H. (2004). Convex approximations for complete integer recourse models. Mathematical Programming, 99(2), 297-310.

Wallace, S. W., \& Ziemba, W. T. (Eds.) (2005). Series on optimization. MPS-SIAM series in optimization. Applications of stochastic programming. Philadelphia: SIAM.

Zenios, S. A., \& Ziemba, W. T. (Eds.) (2006). Handbooks in finance: Vol. 1. Handbook of asset and liability management: theory and methodology. Amsterdam: Elsevier.

Ziemba, W. T., \& Mulvey, J. M. (Eds.) (1998). World wide asset and liability management. Cambridge: Cambridge Univ. Press. 